Journal of Animal and Veterinary Advances 10 (17): 2208-2214, 2011

ISSN: $1680-5593$

(C) Medwell Journals, 2011

\title{
Estimation Response to Selection in Three Commercial Lines of Silkworm (Bombyx mori L.) Having Peanut Cocoon
}

\author{
${ }^{1}$ Reza Neshagaran Hemmatabadi, ${ }^{2}$ Alireza Seidavi and ${ }^{1}$ Shahaboddin Gharahveysi \\ ${ }^{1}$ Department of Animal Science, Islamic Azad University, Qaemshahr Branch, Qaemshahr, Iran \\ ${ }^{2}$ Department of Animal Science, Islamic Azad University, Rasht Branch, Rasht, Iran
}

\begin{abstract}
The records and responses to selection were analyzed in 4 generations of three pure and commercial lines of 31, 103 and 107 Iranian silkworm. In the beginning year of experiment, a parental group was selected, recorded and coded for each 3 lines of 31, 103 and 107 which had further weight average than the population average. These parents were crossed with each other and a selected group was made for these three lines. Also a parental group was chosen, recorded and coded for each of the three lines which include the weight average equal to population average weight. These parents also were crossed with each other and a coincidental group was made for these three lines. The members of each group were grown up and crossed during three successive generations coincidentally with out any re selection. The 27 average records were registered and analyzed. The data analysis in this research showed that among three studied lines in the whole year for larva vitality percentage, the highest response to selection is shown by line $103(28.62 \%)$ then line $31(70 \%)$ and their least line $107(-3.90 \%)$ in the base population. The comparison of average response to these three lines selection also shows that the difference of response to the selection between lines is not significant statistically $(\mathrm{p}>0.05)$. Among lines in the whole year for larva remaining percentage, the highest response to selection was observed in line 31 (33.13 cocoon) followed by line 103 (17.91 cocoon) in the base population. Also, the least response to the selection in base population belonged to line 107 (9.29 cocoon). The comparison of average response to these lines selection showed that the response difference to selection in lines has not been significant statistically ( $p>0.05$ ). Among the three studied lines in a whole year for a best cocoon total weight, the highest response to the selection in base population belonged to line 31 (49.44 g) then in line 103 (14.02 g) and the least response to selection in the base population belonged to line $107(10.90 \mathrm{~g})$. The comparison of average response to these three lines selection showed that there was not any statistical meaning in response difference to the selection among them ( $>0.05$ ). Among lines in the whole year for best single cocoons weight, the highest response to the selection in base population belonged to line $31(0.05763 \mathrm{~g})$ and then line 107 $(0.05142 \mathrm{~g})$ and the least response to selection belonged to the line $103(0.014 .7 \mathrm{~g})$ in the base population. The comparison of average response to selection between three lines has shown that the response difference to the selection among lines is not significant statistically $(\mathrm{p}>0.05)$. Through lines in a whole year for the cocoon weight resulted form 10000 larva, the highest response to selection in the base population belonged to line 31 $(601.1 \mathrm{~g})$ then line $107(572.9 \mathrm{~g})$. The least response to the selection was observed in line $103(-41.1 \mathrm{~g})$. The comparison of average response to the selection through lines show that the response difference to the selection among these three lines is not significant statistically $(\mathrm{p}>0.05)$.
\end{abstract}

$\underline{\text { Key words: Peanut cocoon, generation, phonotype, individual selection, lines comparison, Iran }}$

\section{INTRODUCTION}

According to science and technology development in the field of silk production in silk producing regions and exporting countries, these countries have come to this conclusion that the basis and scale of competition in the field of producing raw silk and cocoons is to use the suitable in breeding plan to catch a good various silkworm. In fact, silkworm in breeding is in order to make more cocoon, more suitable and faster solution to find the raw silk and with an excellent quality (Seidavi and Bizhannia, 2008). Every progress in breeding comes in using the breeding methods and selection which depends on recognition and inter coursing the animals which have superior in heritance for a specific aim. So if there is a need to have any progress, it is these superior animals which must make the future generation. This aim of this experiment was the study of three Iranian silkworm lines

Corresponding Author: Alireza Seidavi, Department of Animal Science, Islamic Azad University, Rasht Branch, Rasht, Iran 
(31, 103 and 107) versus response to selection which can use all their genetic capacity to produce in the case of having high genetic capacity in usual management. Then using the crosses between selected parents, there would be better use of them. This can be important and efficient in silkworm management and growing amend. The targets of this test were included in response evaluation to the selection of three silkworm commercial lines in continues generations and the comparison of response to three commercial lines $(107,103$ and 31$)$ of Iran silkworm for different economical traits in total year.

\section{MATERIALS AND METHODS}

This research data was achieved from Iran Silkworm Research Center (ISRC). Kenmochi, Kinese and Shin Ichinose can be named of this region mulberry trees. These three lines of commercial lines have the peanut shape. Two groups were made in each of these three lines, one with the similar average to the population. The inter group intercourses were happened with out reselection during three followed generations, the economical characters of each of two selected group and evidence group were recorded and the response to selecting of each trait was measured from the difference of the average trait in two selected groups and evidence group in the proportion of their previous generation by dividing into lines, group and trait.

The information used in this study, the single dependent records to the important economical traits includes three pure commercial lines of 31, 103 and 107 Iran silkworm. This information was recorded, classified and saved in computer by the Excel software. The considered parameters followed in four generation to compare the methods activity results. The record making of under studied traits also was done according to instruction and standard protocol. After collecting the related data, the variation analysis and average SAS software was used; comparison between studied traits were done. To analyze the variation based on Completely Random Design (CRD) and also to compare the average. The average comparison between traits was also done by Duncan method and in a significant level $(\alpha=5 \%)$.

To do this test, according to related records to the members of the three pure commercial lines of 31,103 and 107 including Iran peanut shaped silkworm cocoons for each of these three lines, a selected group and an evident were studied as the base population. The first one included the members with higher cocoon average weight than that of the population and the later included the member with the average similar to the population average. In the $2 \mathrm{nd}$, 3rd and 4 th of this research, there were in group intercourses with out reselecting then the economical traits were record made in each of two groups of selection and evidence then respond to each trait selection was measured by deferential of trait average in selection group and evidence group in proportion to its previous generation in separation to each line, group and trait. Larva breeding was done by using the wooden tray method. In this method, larvae were fed three times a day according to the standard times. At the time of molting, lime was used to decrease the bed moisture. Respecting the quality, the produced silkworm cocoons divided in to four groups of best cocoons, medium cocoons, low cocoons and double (2 larvae). The amount and percentage of each cocoon group was estimated in proportion to the total produced cocoons.

Studied characters included alive larvae number, alive pupae number, pupae vitality percentage, pupae vitality percentage(best cocoon), pupae vitality percentage (middle cocoon), produced cocoon number, best cocoon number, middle cocoon number, low cocoon number, double cocoon number, best cocoon percentage, middle cocoon percentage, low cocoon percentage, double cocoon percentage, best cocoon weight, double cocoon weight, single best cocoon weight, 10000 larvae cocoon weight, larval duration, hatched larvae un, hatched eggs, unfertilized eggs, hatched eggs percentage, unhatched eggs percentage, unfertilized eggs percentage, hatchability percentage total produced eggs.

\section{RESULTS}

Comparison of three studied lines for individual selection effect based on cocoon weight in zero generation on response to selection of alive larvae number: Data analysis are showed that among three studied lines, the highest response to selection in zero generation is belonged to line 31 (36.96) and 103 (18.57), respectively and line 107 (11.83) remained at lower response to selection than other lines. Meanwhile, statistical differences of response to selection among three studied lines in this character were not significant $(\mathrm{p}>0.05)$.

\footnotetext{
Comparison of three studied lines for individual selection effect based on cocoon weight in zero generation on response to selection of alive pupae number: Data analysis are showed that among three studied lines, the highest response to selection in zero generation is belonged to line 103 (133.4) and 31 (29.6), respectively and line 107 (2.5) remained at lower response to selection than other lines. Meanwhile, statistical differences of response to selection among three studied lines in this character were not significant $(\mathrm{p}>0.05)$.
} 
Comparison of three studied lines for individual selection effect based on cocoon weight in zero generation on response to selection of pupae vitality percentage: Data analysis are showed that among three studied lines, the highest response to selection in zero generation is belonged to line $103(28.62 \%)$ and $31(0.70 \%)$, respectively and line $107(-3.90 \%)$ remained at lower response to selection than other lines. Meanwhile, statistical differences of response to selection among three studied lines in this character were not significant $(\mathrm{p}>0.05)$.

Comparison of three studied lines for individual selection effect based on cocoon weight in zero generation on response to selection of pupae vitality percentage (best cocoon): Data analysis are showed that among three studied lines, the highest response to selection in zero generation is belonged to line $31(1.190 \%)$ and $103(-1.944 \%)$, respectively and line $107(-2.940 \%)$ remained at lower response to selection than other lines. Meanwhile, statistical differences of response to selection among three studied lines in this character were not significant $(\mathrm{p}>0.05)$.

Comparison of three studied lines for individual selection effect based on cocoon weight in zero generation on response to selection of pupae vitality percentage (middle cocoon): Data analysis are showed that among three studied lines, the highest response to selection in zero generation is belonged to line 31 (1.501\%) and $103(-3.947 \%)$, respectively and line $107(-4.849 \%)$ remained at lower response to selection than other lines. Meanwhile, statistical differences of response to selection among three studied lines in this character were not significant $(\mathrm{p}>0.05)$.

Comparison of three studied lines for individual selection effect based on cocoon weight in zero generation on response to selection of produced cocoon number: Data analysis are showed that among three studied lines, the highest response to selection in zero generation is belonged to line 31 (33.13) and 103 (17.91), respectively and line 107 (9.29) remained at lower response to selection than other lines. Meanwhile, statistical differences of response to selection among three studied lines in this character were not significant $(\mathrm{p}>0.05)$.

Comparison of three studied lines for individual selection effect based on cocoon weight in zero generation on response to selection of best cocoon number: Data analysis are showed that among three studied lines, the highest response to selection in zero generation is belonged to line 31 (24.88) and 103 (3.26), respectively and line $107(0.17)$ remained at lower response to selection than other lines. Meanwhile, statistical differences of response to selection among three studied lines in this character were not significant ( $\mathrm{p}>0.05)$.

Comparison of three studied lines for individual selection effect based on cocoon weight in zero generation on response to selection of middle cocoon number: Data analysis are showed that among three studied lines, the highest response to selection in zero generation is belonged to line 103 (15.304) and 107 (7.833), respectively and line 31 (4.583) remained at lower response to selection than other lines. Meanwhile, statistical differences of response to selection among three studied lines in this character were not significant $(\mathrm{p}>0.05)$.

Comparison of three studied lines for individual selection effect based on cocoon weight in zero generation on response to selection of low cocoon number: Data analysis are showed that among three studied lines, the highest response to selection in zero generation is belonged to line 103 (3.043) and 107 (2.208), respectively and line $31(0.167)$ remained at lower response to selection than other lines. Meanwhile, statistical differences of response to selection among three studied lines in this character were not significant ( $\mathrm{p}>0.05)$.

Comparison of three studied lines for individual selection effect based on cocoon weight in zero generation on response to selection of double cocoon number: Data analysis are showed that among three studied lines, the highest response to selection in zero generation is belonged to line 31 (3.500) and 107 (-0.917), respectively and line $103(-3.696)$ remained at lower response to selection than other lines. Meanwhile, statistical differences of response to selection among three studied lines in this character were significant $(\mathrm{p}<0.05)$.

Comparison of three studied lines for individual selection effect based on cocoon weight in zero generation on response to selection of best cocoon percentage: Data analysis are showed that among three studied lines, the highest response to selection in zero generation is belonged to line $31(0.584 \%)$ and $107(-2.493 \%)$, respectively and line $103(-3.086 \%)$ remained at lower response to selection than other lines. Meanwhile, statistical differences of response to selection among three studied lines in this character were not significant $(\mathrm{p}>0.05)$.

Comparison of three studied lines for individual selection effect based on cocoon weight in zero generation on response to selection of middle cocoon percentage: Data analysis are showed that among three studied lines, the 
highest response to selection in zero generation is belonged to line 103 (3.589\%) and 107 (1.976\%), respectively and line $31(-0.263 \%)$ remained at lower response to selection than other lines. Meanwhile, statistical differences of response to selection among three studied lines in this character were not significant $(\mathrm{p}>0.05)$.

Comparison of three studied lines for individual selection effect based on cocoon weight in zero generation on response to selection of low cocoon percentage: Data analysis are showed that among three studied lines, the highest response to selection in zero generation is belonged to line $107(1.278 \%)$ and $103(0.728 \%)$, respectively and line $31(-1.293 \%)$ remained at lower response to selection than other lines. Meanwhile, statistical differences of response to selection among three studied lines in this character were not significant $(\mathrm{p}>0.05)$.

Comparison of three studied lines for individual selection effect based on cocoon weight in zero generation on response to selection of double cocoon percentage: Data analysis are showed that among three studied lines, the highest response to selection in zero generation is belonged to line $31(0.9733 \%)$ and $107(-0.7617 \%)$, respectively and line $103(-1.2313 \%)$ remained at lower response to selection than other lines. Meanwhile, statistical differences of response to selection among three studied lines in this character were significant $(\mathrm{p}<0.05)$.

Comparison of three studied lines for individual selection effect based on cocoon weight in zero generation on response to selection of best cocoon weight: Data analysis are showed that among three studied lines, the highest response to selection in zero generation is belonged to line 31 (49.44 g) and $103(14.02 \mathrm{~g})$, respectively and line 107 (10.90 g) remained at lower response to selection than other lines. Meanwhile, statistical differences of response to selection among three studied lines in this character were not significant $(\mathrm{p}>0.05)$.

Comparison of three studied lines for individual selection effect based on cocoon weight in zero generation on response to selection of double cocoon weight: Data analysis are showed that among three studied lines, the highest response to selection in zero generation is belonged to line $31(0.0412 \mathrm{~g})$ and $103(-0.1350 \mathrm{~g})$, respectively and line $107(-0.1654 \mathrm{~g})$ remained at lower response to selection than other lines. Meanwhile, statistical differences of response to selection among three studied lines in this character were not significant $(\mathrm{p}>0.05)$.

Comparison of three studied lines for individual selection effect based on cocoon weight in zero generation on response to selection of single best cocoon weight: Data analysis are showed that among three studied lines, the highest response to selection in zero generation is belonged to line $31(0.05763 \mathrm{~g})$ and $107(0.05142 \mathrm{~g})$, respectively and line $103(0.01417 \mathrm{~g})$ remained at lower response to selection than other lines. Meanwhile, statistical differences of response to selection among three studied lines in this character were not significant $(\mathrm{p}>0.05)$.

Comparison of three studied lines for individual selection effect based on cocoon weight in zero generation on response to selection of 10000 larvae cocoon weight: Data analysis are showed that among three studied lines, the highest response to selection in zero generation is belonged to line 31 (601.1 g) and 107 (572.9 g), respectively and line $103(-41.1 \mathrm{~g})$ remained at lower response to selection than other lines. Meanwhile, statistical differences of response to selection among three studied lines in this character were not significant $(\mathrm{p}>0.05)$.

Comparison of three studied lines for individual selection effect based on cocoon weight in zero generation on response to selection of larval duration: Data analys is are showed that among three studied lines, the highest response to selection in zero generation is belonged to line $103(1.652)$ and $31(-0.542)$, respectively and line $107(-3.333)$ remained at lower response to selection than other lines. Meanwhile, statistical differences of response to selection among three studied lines in this character were significant $(\mathrm{p}<0.05)$.

Comparison of three studied lines for individual selection effect based on cocoon weight in zero generation on response to selection of hatched larvae: Data analysis are showed that among three studied lines, the highest response to selection in zero generation is belonged to line 103 (50.57) and 107 (26.25), respectively and line 31 (8.38) remained at lower response to selection than other lines. Meanwhile, statistical differences of response to selection among three studied lines in this character were not significant $(\mathrm{p}>0.05)$. 
Comparison of three studied lines for individual selection effect based on cocoon weight in zero generation on response to selection of unhatched eggs: Data analysis are showed that among three studied lines, the highest response to selection in zero generation is belonged to line $103(-0.174)$ and $31(-2.375)$, respectively and line 107 $(-3.333)$ remained at lower response to selection than other lines. Meanwhile, statistical differences of response to selection among three studied lines in this character were not significant $(\mathrm{p}>0.05)$.

Comparison of three studied lines for individual selection effect based on cocoon weight in zero generation on response to selection of unfertilized eggs: Data analysis are showed that among three studied lines, the highest response to selection in zero generation is belonged to line 103 (7.043) and $107(1.917)$, respectively and line $31(-13.375)$ remained at lower response to selection than other lines. Meanwhile, statistical differences of response to selection among three studied lines in this character were significant $(\mathrm{p}<0.05)$.

Comparison of three studied lines for individual selection effect based on cocoon weight in zero generation on response to selection of hatched eggs percentage: Data analysis are showed that among three studied lines, the highest response to selection in zero generation is belonged to line 31 (2.303) and $107(0.675)$, respectively and line $103(-2.200)$ remained at lower response to selection than other lines. Meanwhile, statistical differences of response to selection among three studied liness in this character were not significant $(\mathrm{p}>0.05)$.

Comparison of three studied lines for individual selection effect based on cocoon weight in zero generation on response to selection of unhatched eggs percentage: Data analysis are showed that among three studied lines, the highest response to selection in zero generation is belonged to line $31(-0.0054 \%)$ and $107(-0.7100 \%)$, respectively and line $103(-0.7778 \%)$ remained at lower response to selection than other lines. Meanwhile, statistical differences of response to selection among three studied lines in this character were not significant $(\mathrm{p}>0.05)$.

Comparison of three studied lines for individual selection effect based on cocoon weight in zero generation on response to selection of unfertilized eggs percentage: Data analysis are showed that among three studied lines, the highest response to selection in zero generation is belonged to line $103(0.976 \%)$ and $107(0.035 \%)$, respectively and line $31(-2.296 \%)$ remained at lower response to selection than other lines. Meanwhile, statistical differences of response to selection among three studied lines in this character were significant $(\mathrm{p}<0.05)$.

Comparison of three studied lines for individual selection effect based on cocoon weight in zero generation on response to selection of hatchability percentage: Data analysis are showed that among three studied lines, the highest response to selection in zero generation is belonged to line $107(0.7600 \%)$ and $103(0.7335 \%)$, respectively and line $31(0.1763 \%)$ remained at lower response to selection than other lines. Meanwhile, statistical differences of response to selection among three studied lines in this character were not significant $(\mathrm{p}>0.05)$.

Comparison of three studied lines for individual selection effect based on cocoon weight in zero generation on response to selection of total produced eggs: Data analysis are showed that among three studied lines, the highest response to selection in zero generation is belonged to line 103 (57.57) and 107 (24.83), respectively and line $31(-7.38)$ remained at lower response to selection than other lines. Meanwhile, statistical differences of response to selection among three studied lines in this character were not significant ( $\mathrm{p}>0.05)$.

\section{DISCUSSION}

It was shown in this research that the difference in level ( $p>0.05$ ) had higher meaning for some traits in autumn than those of spring and the whole year. The results got from the Pashaki (2010)'s research on three commercial lines (110, 104 and 32) showed that the average difference of all lines was significant regarding to economical traits of cocoons weight $(\mathrm{p}<0.0 .1)$. This shows that there is a genetic difference in commercial studied lines. The genetic potential of every variance is different regarding to various traits. As a sample when grouping was done based on special groups of traits like best cocoon weight, the results got from dandogram and the related group became different from related grouping by the same but with a live larva group. This caused that various grouping could be observed for 27 traits even in one method.

The basic effect of selection is to change the gene abundance. However, genes abundance changing are almost invisible by themselves because the gene positions effect related to a quantitative trait can not be studied one by one. So, selection effect can be observed 
in population average changes by this way, gene abundant changes are considered a little more and in its total format (Falconer, 1990). Usually, there are three basic factors which influence on progressing the amount of quantitative traits of under selection effect; selection difference, inheritance taking and distance between two generations. Rangaiah et al. (1995) have reported a positive correlation between a cocoon weight, a silk tissue weight and silk tissue percent with the fertile eggs percentage and have emphasized on the importance of these traits in inbreeding programs. Petkov and Nguyenvan (1987) also studied some genetic traits in some new silkworm variances and claimed the correlation between cocoon tissue weight and cocoon tissue percentage as $0.528-0.653$. In addition, the correlation between cocoon tissue weight and silky tissue percentage is greatly significant (Kumar et al., 1995).

Kumar et al. (1995) showed that there is a high correlation between cocoon weight and cocoon tissue and also between cocoon weight and cocoon tissue percentage. In some separated research, a high genetic correlation was reported between cocoon traits (Sofi et al., 1999; Jayaswal et al., 2000). In their research, Mirhosseini et al. (2002) estimated the genetic correlation between cocoon weight and cocoon tissue weight as 0.709-0.989 and the genetic correlation between cocoon weight and cocoon tissue percentage as -0.124-0.564. Seidavi (2010) got high correlations for cocoon weight and cocoon tissue weight. These results showed that these two traits are under the influence of high effect genes and common environmental factors. But there was a low genetic correlation between cocoon weight and cocoon tissue percentage and even in some line, it was negative correlation. As a sum, genetic correlation index of these two traits was calculated in the lines sum as 0.039 . Seidavi (2010) reported medium environmental correlation was achieved between cocoon weight and cocoon tissue percentage (0.577). In addition, the achieved phonotypical correlation between cocoon tissue weight and cocoon tissue percentage, 0.546 came out in total. The selective program mentions to use a pure line to improve the function and economical progress. So, selection has an important role in genetic improvement (Seidavi et al., 2009). Mu et al. (1995) have claimed that inheritance taking in specific concept, specifically meant cocoon tissue weight, cocoon tissue percentage and the weight of 10000 larvae is more than the other traits, in their studies, Malik (1992) also achieved inheritance taking of $0.914,0.779$ and 0.457 for single cocoon weight, cocoon tissue weight and cocoon tissue percentage. Mirhosseini et al. (2005) estimated inheritance taking for cocoon weight as $0.374-0.500$, for cocoon tissue weight as
$0.366-0.725$ and for cocoon tissue percentage as $0.142-0.337$. They also estimated genetic parameters of economical traits for native silkworm in 2005 and showed that inheritance taking is high for economical traits (Mirhosseini et al., 2005). Kumaresan et al. (2007) achieved inheritance taking in general concept for cocoon weight, cocoon tissue weight and cocoon tissue percentage in order $0.4652,0.565$ and 0.3731 by analyzing the change ability in 85 silkworm races of India hot regions.

\section{CONCLUSION}

In the whole year, in line 31,14 more traits, in 4 the least trait and in 4 the average trait responses to selection were observed. In line 103, 11 more traits, in 7 the least trait and in 4 average trait responses to selection were observed. Also, more function in response to selection was observed in line 31 and the least function was observed in line 107, the average function was in line 107 that was the average of two lines of 31 and 107. As a result, the most responses to selection in this research relate to line 31 and the least response to selection relates to line 107 .

\section{REFERENCES}

Falconer, D.S., 1990. Introduction to Quantitative Genetics. 3rd Edn., John Wiley and Sons Inc., New York, pp: 438.

Jayaswal, K.P., S. Masilamani, V. Lakshmanan, S.S. Sindagi and R.K. Datta, 2000. Genetic variation, correlation and path analysis in mulberry, Bombyx mori. Sericologia, 40: 211-223.

Kumar, P., R. Bhutia and M.M. Ahsan, 1995. Estimates of genetics variability for commercial quantitative traits and selection indices in bivoltine races of mulberry silkworm, Bombyx mori L. J. Indian J. Genet. Plant Breed., 55: 109-116.

Kumaresan, P., R.K. Sinha and S.R. Urs, 2007. An analysis of genetic variation and divergence in Indian tropical polyvoltine silkworm (Bombyx mori L.) genotypes. Caspian J. Environ. Sci., 5: 11-17.

Malik, M.A., 1992. Studies on the performance and adaption of bivoltine race of silkworm Bombyx mori L. pf Kashmir and evaluation of hetrosis in their hybrids under temperate and subtropical climates. Ph.D. Thesis, University of Mysore, Mysore, India.

Mirhosseini, S.Z., M. Ghanipoor, M.R. Gholami and A. Shadparvar, 2002. Estimation of genetic parameters for cocoon traits in six Iranian commercial lines. Proceedings of the 15th Iranian Plant Protection Congress, Sept. 7-11, Razi University of Kermanshah, Iran, pp: 281-281. 
Mirhosseini, S.Z., M. Ghanipoor and A.R. Seidavi, 2005. Study of genetic trend of biological and quantitative properties of silkworm in successive generationsin at non-randomized matings populations. Proceeding of the 2nd Animal Science Congress, Sept. 4-5, Guilan University, pp: 111-111.

Mu, Z.M., Q.X. Liu, X.L. Liu, W.G. Li and Z.Y. Sun, 1995. Genetic research of vitality and cocoon quality traits of silkworm. J. Shandong Agric. Univ., 26: 157-163.

Pashaki, A.S., 2010. Genetical parameters estimation of three commercial lines having oval cocoon in Iran. M.A. Thesis, Qaemshahr Branch, Islamic Azad University.

Petkov, N. and L. Nguyenvan, 1987. Breeding genetic studies on some lines of the silkworm, Bombyx mori L. Genetika-i- Selektsiya, 20: 384-354.

Rangaiah, S., R. Govindan, M.C. Devalah and T.K. Narayanswamy, 1995. Genetic studied for some quantitative traits among multivoltine races of silkworm, Bombyx mori L. J. Agric. Sci., 29: 248-251.
Seidavi, A. and A. Bizhannia, 2008. Principles and Techniques of Silkworm Breeding. 1st Edn., Haghshenass Publishing, Iran, pp: 150.

Seidavi, A., Z. Mirhosseini, M. Mavvajpour, M. Ghanipoor, A. Bizhannia, A. Qotbi and M. Chamani, 2009. Additive genetic variations and selection index changes of economic traits of the silkworm commercial pure lines against parent selection pressure. Am. Eurasian J. Agric. Environ., 6: $460-665$.

Seidavi, A., 2010. Estimation of genetic parameters and selection effect on genetic and phenotype trends in silkworm commercial pure lines. Asian J. Anim. Vet. Adv., 5: 1-12.

Sofi, A.M., M.A. Masoodi and A.S. Kamili, 1999. Estimation of heritability and correlation of some quantitative traits in line $\times$ tester analysis in silkworm, Bombyx mori L. Mysore J. Agric. Sci., 33: 289-296. 